\title{
Los modelos de adopción de tecnologías de la información desde el paradigma actitudinal
}

\section{Models of adopting information technologies from the attitudinal paradigm}

Luis Miguel López-Bonilla ${ }^{1}$

Jesús Manuel López-Bonilla²

\section{Resumen}

La difusión de innovaciones es un tópico relevante en la literatura del comportamiento del consumidor. En marketing, hay dos perspectivas que caracterizan a la mayoría de los estudios realizados sobre esta materia: una perspectiva agregada y una perspectiva individual. Los modelos analíticos y empíricos para describir la difusión de una innovación han abarcado el mercado de forma agregada, recibiendo poca atención los modelos de adopción individual. Este trabajo intenta revisar estos modelos de adopción en el ámbito de las TICs.

Palabras claves: modelos de adopción, innovaciones, tecnologías de la información, decisiones del consumidor.

\begin{abstract}
The diffusion of innovations is an important topic in the literature on consumer behaviour. In marketing, two perspectives characterize most of the work on this topic: the aggregate market perspective and the individual-level perspective. The analytical and empirical models for describing and forecasting the diffusion of an innovation have addressed the market in the aggregate, with little attention to the micro-level that characterizes the adoption decision. This paper seeks to revise these models of adoption in the field of IT.
\end{abstract}

Keywords: models of adoption, innovations, Information Technology, consumer decisions.

\footnotetext{
${ }^{1}$ Doctor en Ciencias Económicas y Empresariales por la Universidad de Sevilla; Profesor Titular de la Universidad de Sevilla, Dirección: Facultad de Ciencias Económicas y Empresariales. Endereço: Avda Ramón y Cajal, 41018 Sevilla, España. Email luismi@us.es

${ }^{2}$ Doctor en Ciencias Económicas y Empresariales por la Universidad de Sevilla; Profesor Titular de la Universidad de Sevilla. Dirección: Facultad de Ciencias Económicas y Empresariales. Endereço: Avda Ramón y Cajal, 41018 Sevilla, España. Email lopezbon@us.es
} 


\section{Introducción}

La adopción y difusión de innovaciones es un tópico relevante en la literatura sobre el comportamiento del consumidor. Dentro de la investigación sobre este tópico, se distinguen dos tipos de modelos, como advierten Sinha y Chandrashekaran (1992). Un primer tipo son los modelos que intentan aumentar la comprensión del proceso de difusión en su conjunto. Estos modelos son representaciones analíticas de un proceso de difusión a nivel agregado. A menudo se les conoce como modelos de difusión. Y una segunda clase de modelos tiene el objetivo de aportar claridad respecto a los factores que determinan la decisión de adopción (o no adopción) del individuo. Estos modelos toman una perspectiva desagregada y son conocidos generalmente como modelos de adopción (FRAMBACH et al., 1998). Así, se puede decir que la adopción de un producto es el proceso por el cual un consumidor empieza a comprar y a usar un nuevo bien, servicio o idea. En cambio, la difusión del producto describe cómo se extiende el uso del mismo a través de una población determinada.

El proceso de adopción ha sido definido como el proceso a través del cual los adoptantes individuales pasan desde la concienciación a la aceptación completa de un nuevo producto, procedimiento o idea (OZANNE; CHURCHILL, 1971; ROGERS, 1983). Las decisiones de adopción de innovaciones se diferencian de otros tipos de toma de decisiones por la novedad relativa de estas innovaciones y la mayor incertidumbre asociada a este tipo de decisión.

Se han desarrollado y utilizado numerosos modelos de procesos de adopción en la literatura, a pesar de lo cual, hay quien sostiene que existe poca evidencia empírica que apoye a cualquiera de los modelos propuestos (OLSHAVSKY; SPRENG, 1996). Entre los autores pioneros en la materia, y probablemente más conocidos, destaca Rogers, quién en 1962 adaptó el modelo del proceso de adopción de la literatura de la Sociología con las siguientes cinco etapas: concienciación, investigación, evaluación, prueba y adopción. Ozanne y Churchill (1971) utilizaron el mismo proceso de adopción y lo aplicaron a los productos industriales. Dos décadas después, Rogers (1995) modificó las etapas del proceso de decisión, estructurándolas como sigue: conocimiento, persuasión, decisión, implantación y confirmación. El modelo AIDA (concienciación, interés, deseo y acción) también ha sido usado extensamente en la literatura y en la práctica. Este tipo de modelos ha sido clasificado como modelos positivos por los profesores Antón y Gutiérrez (1997). No obstante, a pesar de la abundante literatura existente, el presente trabajo va a obviar el fenómeno de la adopción de innovaciones desde el punto de vista de este paradigma, inclinándose hacia una aproximación más enraizada en la Psicología que en la Sociología, ya que se adapta mejor a las situaciones de elección individual entre distintas alternativas a la hora de recibir un servicio, donde una de ellas supone el contacto con una tecnología.

De manera general, se puede decir que todos los modelos de adopción de innovaciones tienen tres fases subyacentes: cognitiva, afectiva (evaluativa) y de comportamiento (conativa). En la fase cognitiva el consumidor considera mentalmente o se expone al producto, servicio o idea. Así, el consumidor desarrolla un conjunto de opiniones o creencias sobre las características del producto. Estas ideas se usan para formar sentimientos hacia el producto (fase afectiva). Basándose en los sentimientos, el consumidor emprende las acciones en la fase de comportamiento. 


\section{Modelos cognitivos frente a modelos de actitudes}

La mayoría de los modelos de toma de decisiones y de elección se concentran en los procesos cognitivos, que están basados en el paradigma del procesamiento de la información desarrollado por la Psicología Cognitiva (DABHOLKAR, 1994). Los modelos encuadrados en este paradigma asumen que los individuos reciben información continua de su entorno y procesan esta información para tomar decisiones. Los individuos tienen ciertas reglas mediante las que procesan y manipulan la información, que les sirven para especificar sus procesos de decisión (BETTMAN; JONES, 1972), donde la memoria juega un papel central en todas las fases de procesamiento e interpretación de la información (MALTER, 1996). De acuerdo con este paradigma, las personas procesan la información y crean una representación cognitiva de los conceptos y objetos que pueden ser usados como factores para la elección (LACHMAN; LACHMAN; BUTTERFIELD, 1979). Cuando dos o más conceptos u objetos son comparados y juzgados, los razonamientos se basan en las similitudes, en las diferencias y en las expectativas de utilidad (HAGERTY; AAKER, 1984); la elección ocurre cuando se toma una decisión entre cursos de acción o no acción alternativos (EINHORN; HOGART, 1981), tomando como referencia, principalmente, aquellos atributos comunes a los objetos que son comparados en condiciones de baja implicación ${ }^{3}$ (ZHANG; MARKMAN, 1998, 2001) y los atributos únicos cuando es elevada la motivación del consumidor en el proceso de decisión (ZHANG; MARKMAN, 2001).

Best (2001) distingue, dentro de la Psicología Cognitiva, dos enfoques principales: el paradigma del procesamiento de la información y la teoría conexionista. El primero de ellos puede ser representado mediante la denominada "metáfora computacional", tomando como un símil del proceso mental de una persona a la organización modular de un ordenador, donde el procesamiento de la información es en serie y de manera secuencial. Frente a este enfoque se halla la teoría conexionista, que considera que el procesamiento de información de un individuo debe producirse en paralelo y no en serie, y toma como forma de representación a la "metáfora cerebral", recurriendo en gran número de ocasiones a principios matemáticos y neuronales en sus modelos (BEST, 2001). La teoría conexionista, también denominada de procesamiento paralelo de la información, supone un marco conceptual relativamente reciente en las investigaciones sobre los procesos cognitivos $^{4}$. Además, los investigadores de este paradigma suelen aceptar la "metáfora computacional" como una buena aproximación a la macroestructura del pensamiento, no así para explicar la microestructura (MARTIN; KIECKER, 1990). De esta manera, a los modelos cognitivos se les ha achacado el haber ignorado tradicionalmente el papel de la afectividad, esto es, los aspectos emocional y sentimental en la toma de decisiones del consumidor (BETTMAN; SUJAN, 1987; BAGOZZI, 1990).

Por otro lado, a pesar de que las investigaciones provenientes de la Psicología Social han hecho progresos para comprender el comportamiento del consumidor examinando los procesos cognitivos y afectivos, la principal limitación de esta corriente de investigación es que no consiguen incorporar la elección en los modelos de predicción del comportamiento (AJZEN; FISHBEIN, 1980; SHEPPARD; HARTWICK; WARSHAW, 1988). En otras palabras, la mayoría de los modelos actitudinales, provenientes de la psicología social, se han centrado en un comportamiento dado y se han olvidado de incorporar la elección entre dos o más comportamientos alternativos o cursos de acción (BAGOZZI, 1990). No obstante, hay excepciones a esta

${ }^{3}$ La implicación puede ser definida como la relevancia personal (PETTY; CACIOPPO; SCHUMANN, 1983) o como los sentimientos subjetivos sobre la importancia del proceso de decisión o del objeto sobre el que se realiza el juicio (MANTEL; KARDES, 1999).

${ }^{4}$ Parece surgir a partir de trabajos que consideran las evidencias bioneurológicas sobre el modo de operar del cerebro, que procesa muchas tareas al mismo tiempo (SHEPHERD, 1979; CRICK; ASANUMA, 1986). 
separación en la literatura de los procesos motivacional y de elección, como es el caso de un artículo conceptual de Bagozzi y Van Loo (1991), que examina el papel de las motivaciones y de los valores esperados ${ }^{5}$ en la elección del consumidor, así como otros trabajos cuyos objetivos han sido unificar la corriente actitudinal con la del procesamiento de la información (ZAJONC; MARKUS, 1982; DABHOLKAR, 1991).

\section{Modelos de valores esperados frente a modelos normativos}

En el estudio de los procesos de adopción relacionados con la tecnología se han aplicado dos tipos de modelos: modelos normativos y modelos de los valores esperados (BAGOZZI, 1990). Cada uno de estos modelos se asienta en una de las tradiciones de la literatura de la psicología cognitiva y la psicología social, respectivamente.

Una de las aproximaciones más influyentes para modelizar la adopción de productos tecnológicos es el llamado modelo normativo (HAUSER; URBAN, 1977; TYBOUT; HAUSER, 1981), donde el consumidor selecciona la alternativa que le proporciona mayor utilidad objetiva, siendo ésta determinada habitualmente mediante un procedimiento compensatorio entre las expectativas de ganancia y los costes en los que se puede incurrir (HAGERTY; AAKER, 1984), por lo que los elementos centrales de los modelos normativos son la racionalidad y la optimización (EINHORN; HOGARTH, 1981). De forma gráfica, se puede ver que un estímulo físico, tal como los atributos de un producto, suponen una primera influencia sobre las percepciones que, después, determinan un afecto o preferencia por los productos, conduciendo a la elección.

El otro grupo de modelos es el denominado como modelos de Valores Esperados, que también se conocen como modelos de Actitudes Multiatributo, Compensatorios Lineales o de Fishbein. Los modelos de los valores esperados tienen una larga tradición en la investigación de marketing. La hipótesis subyacente es que una actitud hacia un objeto (o acción) resulta una función de sus creencias acerca de los atributos del objeto (o consecuencias de la acción) y su evaluación de las reacciones afectivas hacia los atributos individuales (o consecuencias).

Las fuentes teóricas del modelo de los valores esperados las encontramos en la teoría de la utilidad esperada subjetiva (v. gr., EDWARDS, 1954) y las teorías de la psicología social (v. gr., FISHBEIN; AJZEN, 1980). Junto a su simplicidad y su sentido común, el modelo de los valores esperados ofrece la ventaja de acomodar tanto los elementos cognitivos como los motivacionales del comportamiento de compra. Una actitud positiva se obtiene sólo cuando las creencias y las evaluaciones son altas al mismo tiempo. En este sentido, el modelo de los valores esperados supera una de las limitaciones principales de los modelos normativos. A pesar de las críticas que ha recibido, el modelo sobrevive y actualmente es aplicado en numerosos trabajos.

${ }^{5}$ Los valores esperados son modelos actitudinales. 


\section{Modelos de adopción de tecnologías de la informaión}

Una vez que se han descrito las diferencias teóricas entre los paradigmas cognitivos y actitudinales, vamos a centrarnos en su aplicación en el campo de las nuevas tecnologías de la información desde la perspectiva de la Psicología Social. Se van a comentar los principales modelos actitudinales utilizados específicamente para investigar la evaluación y adopción de sistemas de información. La mayoría de estos modelos intentan medir la calidad de los sistemas y la evaluación subjetiva de su utilidad para los usuarios.

Los modelos de aceptación de tecnología pueden verse como aplicaciones especializadas de la Teoría de la Acción Razonada (FISHBEIN; AJZEN, 1975, 1980). En el área de los sistemas de información, las actitudes son entendidas como el resultado de las creencias acerca de las características percibidas del sistema. Es importante hacer notar que los modelos actitudinales que vamos a presentar se centran fundamentalmente en los beneficios de los sistemas de información, olvidándose en parte de las expectativas de utilidad negativa derivados de la utilización de dichos sistemas. Los modelos se basan en describir los procesos de información que conducen a las intenciones de aceptar o rechazar una tecnología. Las actitudes hacia un objeto o hacia la realización de un comportamiento se forman tras un proceso de evaluación consciente. Este proceso de evaluación subjetiva ha sido descrito unas veces de manera relativamente simple (con pocos criterios) y otras veces de forma compleja (con muchos criterios e iteraciones). Las evaluaciones están basadas en las percepciones de los objetos y en las consecuencias esperadas de la utilización de dichos objetos. Las consecuencias esperadas se apoyan en las creencias del comportamiento, los conocimientos y, algunas veces, el afecto. Algunos modelos intentan ser puramente cognitivos y otros incluyen medidas de las emociones y de los sentimientos. Así, por ejemplo, Davis (1989) formuló el Modelo de Aceptación de la Tecnología como un proceso cognitivo y desarrolló un modelo similar tres años después, incluyendo medidas de motivación intrínseca como el divertimiento (DAVIS; BAGOZZI; WARSHAW, 1992). También se puede argumentar que el modelo de Moore y Benbasat $(1991,1996)$ y las nuevas versiones del Modelo de Aceptación de la Tecnología (TAM2) (VENKATESH; DAVIS, 2000) incluyen medidas de afecto relacionadas con la imagen.

\section{El modelo de aceptación de la tecnología (TAM)}

El Modelo de Aceptación de la Tecnología fue diseñado para realizar medidas evaluadoras de la calidad de los sistemas de información y de su adaptación a las necesidades del trabajo y, por lo tanto, se utiliza para hacer predicciones de aceptación y uso de nuevas tecnologías. Se mantiene en la línea de la tradición de investigaciones previas de los sistemas de información (SWANSON, 1974; ZMUD, 1978; LARCKER; LESSIG, 1980) que buscan la identificación de los atributos que conducen al éxito de los sistemas de información en la empresa, tomando como medida la satisfacción de los usuarios.

El modelo TAM está basado en la Teoría de la Acción Razonada y en su metodología de los valores esperados. Los autores del modelo ya habían utilizado previamente esta teoría en algunas de sus investigaciones. Así, Bagozzi (1981) lo hizo en un trabajo sobre la donación de sangre, Warshaw y Davis (1984) para una variedad de actividades y Warshaw (1980) en la selección de marcas.

Los estudios basadas en la metodología que propone la Teoría de la Acción Razonada deben adaptarse específicamente a las características del contexto y de la muestra, por lo que los instrumentos utilizados en las encuestas y en las investigaciones ofrecen poca posibilidad de generalizarse a otras muestras y poblaciones, teniendo que generarse nuevos ítems al inicio de cada investigación. Además, la necesidad de crear creencias particulares para cada estudio (entre cinco y doce creencias), conforme a la metodología que usa la Teoría de la 
Acción Razonada, es uno de los mayores inconvenientes de este modelo. Por ello, Davis (1989) se preocupó de buscar unas variables estables que se utilizaran en cualquier situación ${ }^{6}$.

El Modelo de Aceptación de la Tecnología se hizo popular a raíz de que sus escalas de medida predecían la aceptación de los usuarios mostrando unas propiedades psicométricas fuertes, se adaptaba a las necesidades de las investigaciones sobre sistemas de información y era muy fácil de administrar (un total de 21 items). Aunque quizás lo más importante sea que ha permitido la comparación sin complicaciones de diferentes sistemas de información.

El modelo TAM sostiene que la actitud hacia el uso de un sistema de información está basada en dos variables antecedentes, como son la utilidad percibida y la facilidad de uso percibida del sistema. Esto es similar al resultado de los juicios de Bandura (1982) y su concepto de autoeficacia. La utilidad percibida se define como "el vaticinio de un usuario acerca de la probabilidad subjetiva de que utilizando una aplicación específica de un sistema de información se incrementará su rendimiento en una organización" (DAVIS, 1989). Los items incluyen medidas para saber cómo los sistemas podrían permitir al usuario acometer tareas más rápidamente, incrementar la productividad, aumentar la eficiencia y mejorar el rendimiento del trabajo.

Figura 1. Modelo de Aceptación de la Tecnología (TAM)

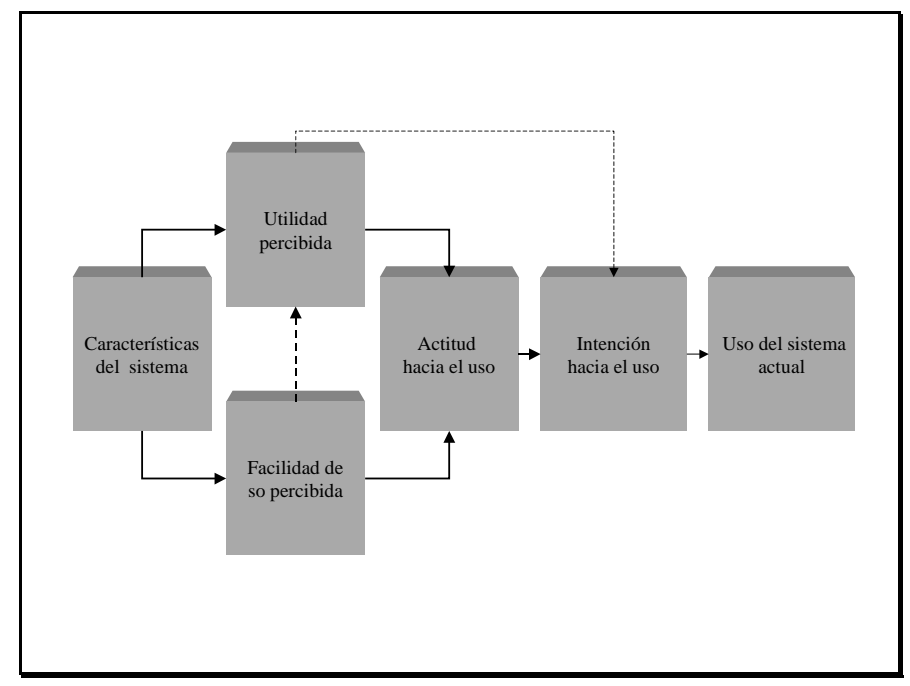

Fuente:Davis 1989

El constructo utilidad percibida está basado en los estudios sobre las motivaciones, las expectativas y las investigaciones de los sistemas de información realizados, entre otros autores, por Vroom (1964), Larcker y Lessig (1980) y Bandura (1982). Esta variable se centra en la habilidad del sistema de información para facilitar al trabajador un rendimiento aceptable y deseable que le permita conseguir, a menudo, recompensas laborales.

\footnotetext{
${ }^{6}$ Davis, Bagozzi y Warshaw (1989) manifiestan: "El objetivo del TAM es proporcionar una explicación de los determinantes de la aceptación de la informática en general, capaz de explicar el comportamiento de los usuarios a través de un amplio abanico de tecnologías informáticas y poblaciones de usuarios". Las variables del TAM están basadas en "un conjunto de creencias que podamos generalizar fácilmente a los diferentes sistemas y poblaciones de usuarios".
} 
Los items que emplea Davis (1989) como indicadores de la utilidad percibida se orientan a conocer las evaluaciones de las personas acerca de las consecuencias que puede tener en la productividad de su trabajo el uso de un determinado sistema de información.

Otro de los constructos fundamentales del TAM es la facilidad de uso percibida de una tecnología, que está basado en la autoeficacia de Bandura (1982), y se define por Davis (1989) como "el grado en el que el usuario espera que el manejo de un determinado sistema conlleve la realización de menores esfuerzos". Los items que miden a este concepto son la flexibilidad, la facilidad de su uso, el control y la dificultad para convertirse en un experto en su uso.

En el Modelo de la Aceptación de la Tecnología (TAM) se propone la vinculación directa entre una de las creencias (la utilidad percibida) y las intenciones (ver figura 1). Esto supone una diferencia significativa respecto a la Teoría de la Acción Razonada, en la que las creencias sólo impactan en las actitudes. Davis (1989), al igual que Triandis (1971), entiende que las actitudes tienen elementos de afectividad y argumenta que la relación directa entre la utilidad percibida y la intención está basada en reglas de decisión cognitivas para mejorar el rendimiento laboral y no necesariamente tienen por qué activar la afectividad

"Si el afecto no está completamente activado cuando se decide usar un determinado sistema, una actitud podría no estar dispuesta para recibir el impacto de las consideraciones del rendimiento relativo a una intención. Por lo tanto, la relación entre la utilidad percibida y la intención representa un efecto directo, al considerar que la gente forma sus intenciones hacia el uso de un ordenador pensando en cómo mejorará el rendimiento de su trabajo" (DAVIS, 1989).

Estudios posteriores han comparado la Teoría de la Acción Razonada o la Teoría del Comportamiento Planeado con la Teoría de la Aceptación de la Tecnología (v. gr., DAVIS; WARSHAW; BAGOZZI, 1989; BAGOZZI, 1990), concluyendo que ésta última proporciona mejores predicciones. Sin embargo, en otros casos no es así (v. gr., TAYLOR; TODD, 1995; HU; CHAU; SHENG; TAM, 1999). Bagozzi (1990), comparando el TAM con la Teoría de la Acción Razonada, indica ciertas mejoras que ofrece el modelo TAM, como son su mayor parsimonia, la posibilidad de evitar que los efectos individuales de las creencias queden ocultos ${ }^{7}$, su mayor flexibilidad $^{8}$ y generalidad ${ }^{9}$.

Desde un punto de vista teórico y, sobre todo, en el ámbito de aplicaciones laborales, se empezó a dudar del papel moderador que desempeña la variable actitudes hacia el uso de la tecnología. De este modo, Davis (1989), al igual que Triandis (1971), entiende que las actitudes tienen elementos de afectividad y argumenta que la relación directa entre utilidad percibida y la intención está basada en reglas de decisión cognitivas para

\footnotetext{
${ }^{7}$ Una fase en la metodología aconsejada por la Teoría de la Acción Razonada consiste en la agregación del conjunto de creencias individuales, una vez que se han multiplicado por sus respectivas valoraciones, en un único constructo, de ahí que se diga que los efectos individuales pueden quedar ocultos. Sin embargo, el TAM presenta la desventaja en el tratamiento de las creencias que algunos determinantes de las actitudes pueden ser olvidados al estar limitados sólo a dos.

${ }^{8}$ La flexibilidad es entendida en el sentido de que existen más caminos de relación entre las creencias y las intenciones que la linealidad tan estricta que propone la Teoría de la Acción Razonada.

${ }^{9}$ La generalidad se identifica con la idea de que el TAM utiliza las mismas variables en cualquier aplicación, mientras que la metodología propuesta por la Teoría de la Acción Razonada señala que es necesario extraer creencias específicas para cada nueva situación. Utilizando este mismo argumento, y contradiciendo a Bagozzi (1990), se puede decir que el TAM es menos flexible que la Teoría de la Acción Razonada ya que no se adapta a las circunstancias específicas de cada situación como hace ésta última.
} 
mejorar el rendimiento laboral y no necesariamente tienen por qué activar a la afectividad. Así, las medidas de ajuste de la actitud se consideró insuficiente para mantenerla dentro del modelo, por lo que, años más tarde, esta variable se suprimió, quedando la utilidad percibida y la percepción de lo fácil de usar como antecedentes directos de las intenciones (DAVIS; BAGOZZI; WARSHAW, 1992; VENKATESH; DAVIS, 2000). No obstante, López Bonilla et al. (2006) comparan ambas versiones del TAM (con y sin las actitudes) utilizando dos procedimientos de estimación diferentes dentro de la metodología de ecuaciones estructurales y comprueban que el TAM primitivo (es decir, el modelo que incluye las actitudes) proporciona una mayor explicación del fenómeno estudiado en uno de los dos procedimientos utilizados que, precisamente, resulta ser el que ofrece un mejor ajuste global del modelo.

\section{Modelo de Adopción y Uso de la Tecnología (TAU)}

El Modelo de Adopción y Uso de la Tecnología fue creado por Bagozzi (1990), en el que pretende superar a los dos paradigmas contemporáneos más relevantes de la teoría de la adopción aplicada a la tecnología: el modelo normativo y el de los valores esperados. Intenta desarrollar una teoría, que el autor denomina de rango medio, con objeto de huir de los inconvenientes de aplicabilidad que plantean las teorías generales, cuya representación esquemática encontramos en la figura 2. No obstante, parece que su esquema está dentro de la tradición de los modelos actitudinales y que se relaciona directamente con aquellos que se derivan de la Teoría del Comportamiento Planeado desarrollada por Azjen $(1985,1991)$.

Figura 2. Modelo de la adopción y uso de la tecnología

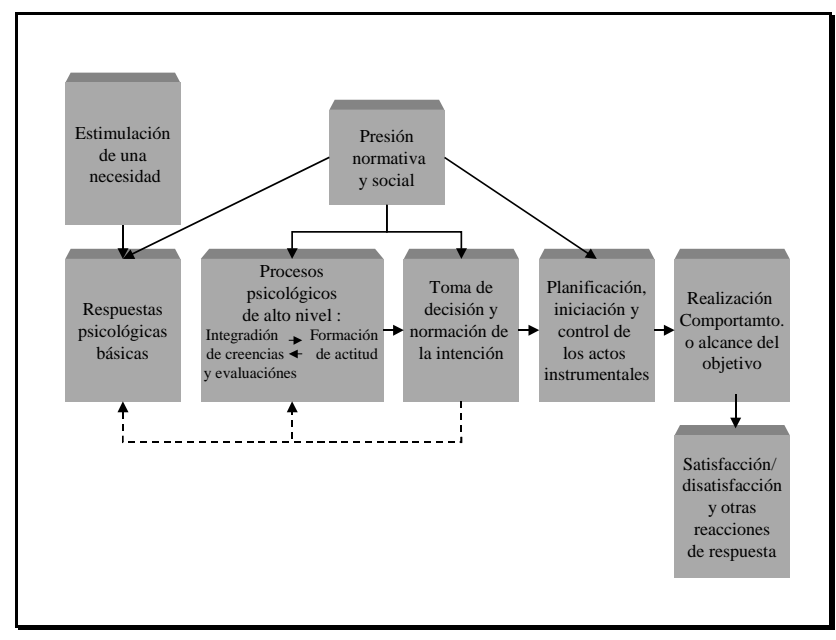

Fuente: Bagozzi (1990)

El proceso comienza con la estimulación de una necesidad, que podría surgir desde dentro de la organización por parte de los tomadores de decisiones (v. gr., reconocimiento de lo inadecuado de un sistema de información), o que podría ser estimulada desde el exterior (v. gr., un vendedor informa a la organización de una necesidad). Seguidamente, se pasa a la fase de respuestas psicológicas básicas, en la que quienes toman decisiones se forman muchas creencias sobre las consecuencias de la adopción del producto y respecto a la puntuación de cada alternativa posible en los atributos del producto. Cada consecuencia y atributo se eligen mediante una evaluación, que supone una primera aproximación de su importancia o valor. 
La motivación para comenzar la valoración se inicia no sólo desde las necesidades propias o de las generadas externamente, sino también de la presión normativa y social dentro de la organización o ejercida desde públicos externos como el gobierno, las asociaciones profesionales, los consumidores u otras organizaciones. Son fuerzas sociales y normativas que influyen sobre la toma de decisiones y las creencias y las evaluaciones que subyacen detrás de estas decisiones. Como se muestra en la figura 2, la influencia social/normativa también comparte estados posteriores en el modelo TAU.

Las creencias originales y las evaluaciones generadas en respuesta a las necesidades o a las oportunidades de nuevos productos están normalmente difusas y desorganizadas, llegando a cristalizarse posteriormente y siendo transformadas a través del estado denominado procesos psicológicos de más alto orden. Aquí se integran las creencias y las evaluaciones y se almacenan como redes de representaciones de valores esperados multidimensionales. Las actitudes hacia los productos alternativos (o hacia proveedores, publicistas, vendedores) y los gustos emergen en esta fase. Algunas veces, las actitudes se desarrollan fuera de las reacciones de los valores esperados, en el sentido de que unas preferencias son guiadas o determinadas por los juicios de los atributos de los productos ponderados por la importancia de estos atributos. Otras veces, las actitudes evolucionan debido inicialmente a condiciones clásicas, aprendizaje operativo, primeras impresiones, prejuicios u otros procesos; y estas actitudes, en suma, suponen el contexto y estructura de las creencias y de las evaluaciones. Así, en la figura 2 se observan dos flechas en una doble dirección entre las creencias y las evaluaciones y la formación de la actitud. Cabe señalar que las actitudes se dividen en tres componentes (hacia el éxito, hacia el fracaso y hacia la persecución del objetivo) de manera similar a la Teoría de la Persecución de una Meta (BAGOZZI; WARSHAW, 1990).

Una vez que se han identificado los criterios de la toma de decisión y de la valoración de los atributos y se han formado las actitudes hacia las alternativas, comienza la fase de toma de decisión y formación de la intención. Respecto a la toma de decisión, se pueden seguir uno o dos cursos de acción. Se debe tomar una elección entre diversas alternativas. $\mathrm{O}$ bien, si no existe ninguna alternativa satisfactoria o si faltan los datos necesarios para tomar una decisión, se procura la vuelta a la información básica reunida y/o a la evaluación. Este último curso se traza con flechas discontinuas en la figura 2. Asumiendo que se toma una decisión, el resultado se expresa de manera volitiva como la intención de comprar un producto concreto.

La mayoría de las decisiones y de las intenciones de actuar, sin embargo, son ejecutadas raras veces de manera fácil o inmediata. Más bien, un amplio conjunto de pasos operativos deben ser reunidos al unísono en un mismo movimiento antes de alcanzar cualquier fin. Bagozzi (1990) llama a esta actividad planificación, iniciación y control de los actos instrumentales. Constituyen los pasos necesarios para transformar una decisión u objetivo en una actuación.

El proceso es consumado a través de los actos físicos y contractuales de la prueba concreta o adopción. Quizás sea menos obvia la idea que señala que el comportamiento puede estar orientado a alcanzar un objetivo, que puede ser instrumental (v. gr., conseguir el uso a un nivel X), o bien, final (v. gr., aumentar la productividad). Se conoce a este estado como realización del comportamiento o alcance del objetivo. Pero el proceso no finaliza aquí. La satisfacción-insatisfacción y otras reacciones de respuesta reajustan la función de cada uno de los estados previos. Por ejemplo, las expectativas son confirmadas o desconfirmadas, las evaluaciones reorientadas, las actitudes cambiadas o consolidadas y las nuevas decisiones reforzadas. 


\section{Modelos de Utilización de las Tecnologías de la Información (UIT) y de Adopción de las Tecnologías de la Información (AIT)}

Moore y Benbasat $(1991,1996)$ desarrollan un modelo para predecir el uso de los ordenadores personales, que supone una innovación en el ámbito donde se realiza el estudio. El Modelo de Utilización de las Tecnologías de la Información (UIT) trata de integrar la Teoría de Difusión de Innovaciones y la Teoría de la Acción Razonada, utilizando las escalas validadas por la Teoría de la Difusión que miden las características percibidas de las innovaciones.

De acuerdo con la Teoría de la Acción Razonada, el uso real de un bien o servicio (tal como un sistema de información) está basado en la actitud hacia su uso. En la teoría de la difusión, las percepciones de la innovación se consideran que son los determinantes más relevantes en la decisión de adoptar o rechazar la innovación. Moore y Benbasat $(1991,1996)$ sitúan a las características percibidas de usar la innovación como los determinantes de la actitud hacia la adopción. Por consiguiente, al contrario que en el modelo de Davis (1989), todas las características percibidas de la innovación son antecedentes directos de la actitud hacia la adopción, sin que aparezcan efectos indirectos, en contradicción a lo que ocurre en el TAM. Otra diferencia fundamental entre estos dos modelos es que en el UIT se incluye un constructo que hace referencia a las normas subjetivas, al igual que en la Teoría de la Acción Razonada.

Para adaptar el Modelo de la Acción Razonada a un contexto de adopción de un sistema de información, se añadió la variable denominada voluntariedad, que modera los efectos de la actitud hacia la adopción y de la variable dependiente clave, que es el uso. En la Teoría de la Acción Razonada es la intención de uso la que cumple este papel moderador.

La adopción de la informática o de los sistemas de comunicación, en muchas organizaciones, está impuesta por la dirección y, por lo tanto, no es un acto volitivo en el que se produzca una elección. Por ello, Moore y Benbasat (1991, 1996) pensaron que la variable voluntariedad cambiaría la importancia relativa de las características percibidas de la innovación. Otro constructo añadido más tarde al modelo es la evasión (IGBARIA, 1993), que es una medida de las fobias y miedos de un adoptante potencial hacia los sistemas de información, dando como resultado una propensión a evitarlo.

Moore y Benbasat (1991) validaron una escala de 39 items para estudiar las características percibidas del comportamiento de uso de la innovación, para lo que tomaron las cinco características percibidas de la innovación desarrolladas por la Teoría de la Difusión de Innovaciones. Sin embargo, ampliaron posteriormente estas cinco características, haciendo que el factor de prestigio social (estatus en la teoría de difusión) fuese separado de la variable ventaja relativa, usándose para formar la variable imagen. Además, la variable observabilidad de la teoría de la difusión fue dividida en dos: visibilidad y demostrabilidad de los resultados.

Agarwal y Prasad (1997a) validaron las características percibidas de las innovaciones propuestas por Moore y Benbasat (1991) como antecedentes del constructo uso habitual del sistema de información, que a su vez influirá en las intenciones futuras de uso. Sin embargo, Agarwal y Prasad (1997b, 1998, 2000), en sus trabajos posteriores, limitan estas características a sólo tres de ellas: ventaja relativa, fácil de usar y compatibilidad. También encontraron apoyo empírico para la variable voluntariedad (AGARWAL; PRASAD, 1997a, 2000), que es utilizada por Moore y Benbasat (1991), en unos casos, como un componente de las percepciones de los usuarios y, en otros (MOORE; BENBASAT, 1996), como variable independiente con una influencia directa sobre las intenciones de uso. 


\section{Figura 3: Modelo de Utilización de la Tecnología de la Información (UIT)}

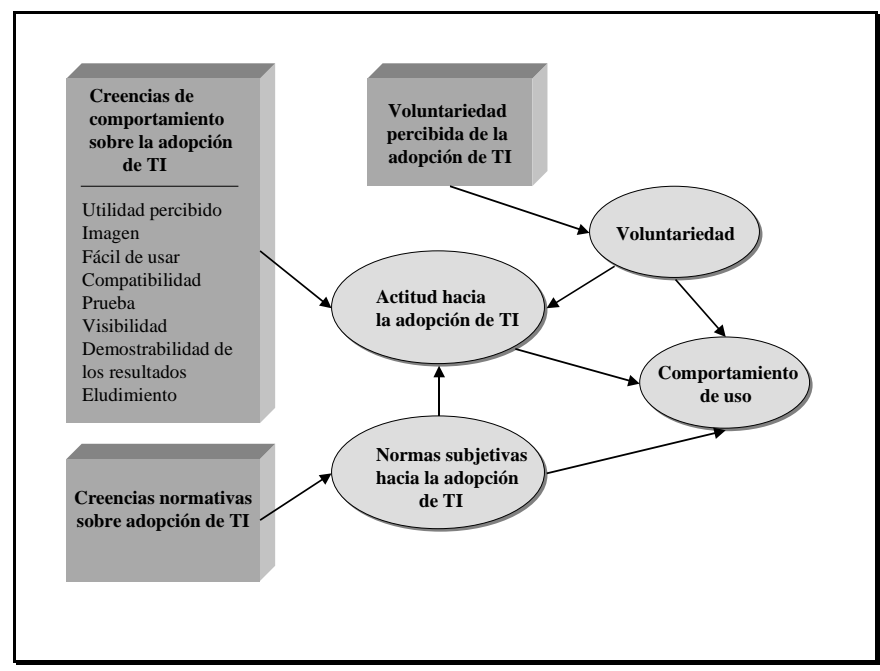

Fuente: Moore y Benbasat (1991; 1996)

Agarwal, Prasad y Zanino (1996) amplían el modelo introduciendo, además del entrenamiento, dos nuevos tipos de variables, situacionales e individuales, que son consideradas como antecedentes de las percepciones de los usuarios, que se identifican con las creencias de comportamiento en el modelo de Moore y Benbasat (1996). Las variables situacionales intentan recoger el contexto de las tareas y de la organización donde el nuevo sistema de información se va a utilizar. Las variables individuales tratan de abarcar las características personales de los potenciales usuarios. Y el entrenamiento trata de recoger la experiencia en el uso de sistemas similares. Agarwal y Prasad (2000) realizan una nueva propuesta donde los determinantes de las intenciones son las actitudes y la voluntariedad. Los de las actitudes se identifican con las creencias ventaja relativa, fácil de usar y compatibilidad, mientras que los determinantes de estas tres son la posición dentro de la plantilla de trabajadores, la inseguridad en el trabajo, los conocimientos técnicos previos y los entrenamientos estructurados (v. gr., mediante clases en sentido tradicional) y sin estructurar (v. gr., a través de sistemas online).

Karahanna, Straub y Chervany (1999) proporcionaron más evidencias empíricas sobre el Modelo de Utilización de la Tecnologías de las Información (UIT). Usaron seis de las siete características percibidas de las innovaciones definidas por Moore y Benbasat $(1996)^{10}$ y la medida de voluntariedad percibida, aplicándola a una investigación diacrónica en la que se compararon las creencias antes de realizar la adopción (evaluaciones de pre-adopción) con otras que se hicieron una vez que se llevó a cabo la adopción (evaluaciones de postadopción). Las principales variables dependientes comparadas fueron las intenciones de realizar el comportamiento con las intenciones de continuar con el uso de la tecnología. Además, se compararon los antecedentes claves de las actitudes hacia los sistemas a adoptar y las actitudes hacia la continuación del uso de los sistemas. El modelo se aprecia en la figura 4.

Sus resultados indican que las intenciones de pre-adopción estaban dominadas por las creencias normativas, mientras que las intenciones de post-adopción lo estaban por las creencias actitudinales. Asimismo, los

${ }^{10}$ Los autores prescindieron de la característica denominada compatibilidad. 
determinantes de las actitudes difieren entre los grupos de pre-adoptantes y post-adoptantes. Todas las características percibidas sobre el uso de la innovación fueron significativas en la pre-adopción, excepto la imagen, y en la fase de post-adopción sólo lo fueron la imagen y la utilidad percibida.

\section{Figura 4: Modelo de Adopción de la Tecnología de la Información}

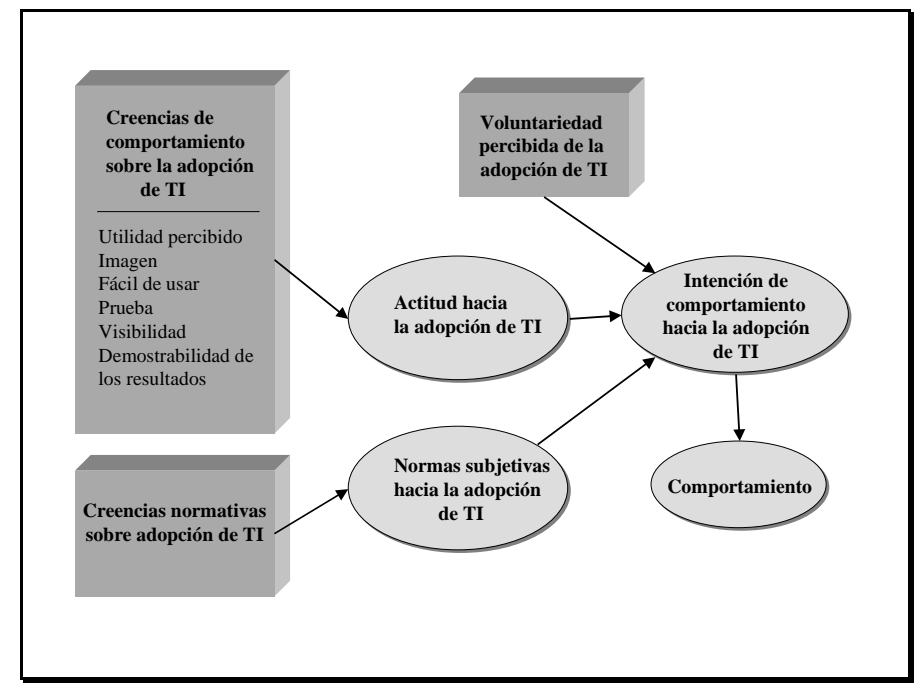

Fonte: Karahanna, Straub y Chervany, (1992)

Tan y Teo (2000), en un trabajo sobre el uso de la banca electrónica, también mezclan la Teoría de Comportamiento Planeado con la Teoría de Difusión de Innovaciones, pero las distintas características de las innovaciones (ventaja relativa, compatibilidad, complejidad, prueba y riesgo ${ }^{11}$ ) no son consideradas como creencias sino como actitudes. En el modelo se incluyen las normas subjetivas y el control percibido, que se divide en dos dimensiones: la autoeficacia y las condiciones facilitadoras. En general, el modelo es estadísticamente significativo, salvo algunas relaciones que no se corroboran, como las influencias de las normas subjetivas, de la complejidad y del apoyo tecnológico ${ }^{12}$ sobre las intenciones de uso.

Los modelos de Utilización de las Tecnologías de la Información (UIT) y de Adopción de la Tecnología de Información (AIT) han proporcionado una vinculación importante entre las tradiciones de investigación más antiguas de la Teoría de la Difusión de Innovaciones y la Teoría de la Acción Razonada y los modelos más actuales como el TAM2.

\section{Modelos de Adopción de la Tecnología derivados del TAM}

El Modelo de Aceptación de la Tecnología (TAM) es probablemente el que goza de un mayor reconocimiento en la literatura sobre adopción en esta última década, siendo no sólo uno de los más citados, sino también un auténtico inspirador de la creación de otros muchos, por lo que cabría la licencia de considerarlos en su mayoría como modelos "hijos" del TAM, "nietos" de la Teoría del Comportamiento Planeado y "biznietos" de la Teoría de la Acción Razonada. No obstante, sólo se va a hacer mención de algunos de estos modelos más destacados.

\footnotetext{
${ }^{11}$ En este modelo la característica riesgo ha sustituido a la observabilidad del modelo de Rogers (1983).

${ }^{12}$ Esta variable forma parte de las condiciones facilitadoras.
} 
En primer lugar hablaremos del Modelo de Motivación Extrínseca e Intrínseca y de la revisión del mismo que practican Davis, Bagozzi y Warshaw (1992). El Modelo de Aceptación de la Tecnología (TAM) está diseñado para predecir el uso de los sistemas informáticos en la empresa. Por ello, el determinante principal para realizar o no la adopción, la utilidad percibida, se centra en aspectos relativos al rendimiento (la productividad y la eficiencia). Tomando como referencia las investigaciones sobre motivación y las taxonomías de la teoría de evaluación cognitiva de Deci (1972), Davis, Bagozzi y Warshaw (1992) identifican a los determinantes del uso de la tecnología relacionados con el rendimiento laboral como motivadores extrínsecos y añaden otros a los que denominan intrínsecos.

La motivación extrínseca se refiere al resultado de una actividad que se percibe como instrumento para alcanzar objetivos valorables, tales como el hecho de que las mejoras de los rendimientos del trabajo pueden ocasionar emolumentos y promociones. La motivación está basada en el valor asignado al reforzamiento (VROOM, 1964; LAWLER; PORTER, 1967).

Figura 5: Modelo de Motivación Extrínseca e Intrínseca

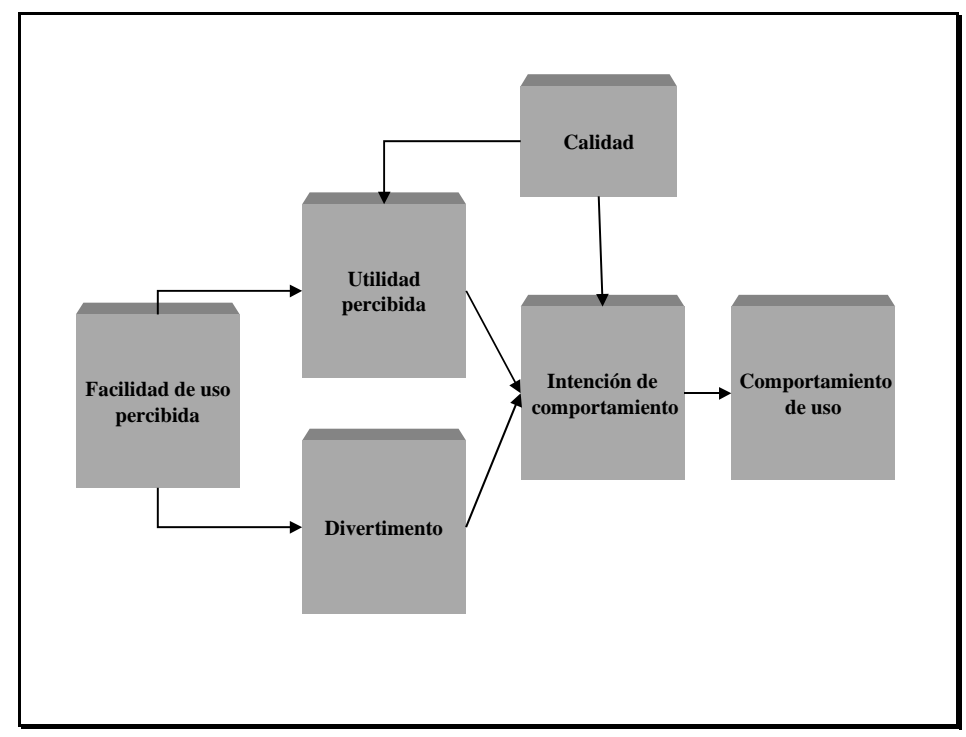

Fuente: Davis, Bargozzi y Warshaw (1992)

La motivación intrínseca conduce a los comportamientos por el puro placer personal y no por una recompensa externa. No se necesario ningún reforzamiento exterior al individuo y el comportamiento se realiza simplemente por su divertimento inherente (DECHARMS, 1968). Muchos comportamientos se hacen por este motivo y los autores del TAM argumentan que el uso de un sistema informático en el lugar de trabajo es uno de ellos. Sostienen que los usuarios valoran el uso de la informática por razones personales más que por la eficiencia organizacional y las recompensas que suponen para el trabajador. "La percepción de lo fácil de usar un sistema y la calidad del mismo también tienen un efecto positivo en el divertimento, ya que representan dos recursos distintos de información relevantes para los sentimientos de autoeficacia, competencia y autodeterminación, que son elementos de la motivación intrínseca" (DAVIS; BAGOZZI; WARSHAW, 1992).

En el estudio realizado por Davis, Bagozzi y Warshaw (1992) se introdujo un segundo modelo denominado Modelo Revisado de Motivación Extrínseca e Intrínseca. En él se empleó una nueva variable: la 
importancia de la tarea a realizar, que moderaba las relaciones entre, por un lado, la facilidad de uso percibida y la utilidad percibida, y, por otro, la calidad y la utilidad percibida. Los resultados de su investigación señalaron que ambas interacciones eran significativas; en cambio, la importancia de la tarea a realizar no era la única variable moderadora de las relaciones indicadas más arriba, ya que también existía un efecto directo entre las variables facilidad de uso percibida y utilidad percibida, así como entre calidad y utilidad percibida. Parece que estos dos modelos suponen un intento de acercamiento del TAM al paradigma motivacional, cuyo éxito al explicar la aceptación de tecnología ha sido muy relevante (VENKATESH; SPEIER, 1999).

Jackson, Chow y Leitch (1997) introdujeron nuevas variables en el TAM y lo denominaron con las siglas TAME (Modelo de Aceptación de Tecnología Extendido), que se representa en la figura 6. Añaden el constructo implicación, propuesto por Zaichowsky (1985) y utilizado por Hartwick y Barki (1994) en la Teoría de la Acción Razonada. Se considera que la implicación del usuario es una variable multidimensional, formada por la implicación situacional y la implicación intrínseca. La primera de ellas se relaciona con la participación de los usuarios en el desarrollo de un sistema de información y es transitoria, tendiendo a cambiar en cada estado del proceso. La segunda se asocia con las metas y los valores de los adoptantes y suele ser estable. Además, se añaden dos constructos que son el uso previo, utilizado por Fredrick y Dossett (1983) en la Teoría de la Acción Razonada, y los argumentos para cambiar, adaptado de Petty, Cacioppo y Schuman (1983) y relacionado con las normas subjetivas de la teoría mencionada, ya que intenta recoger la opinión de los demás sobre los beneficios del sistema. Jackson, Chow y Leitch (1997) concluyen que el modelo TAME explica mejor que el TAM la covarianza entre los diversos constructos y la intención de comportamiento, aunque la varianza explicada por ésta última es similar en los dos modelos.

Figura 6: Modelo de Aceptación de la Tecnología Extendido (TAME)

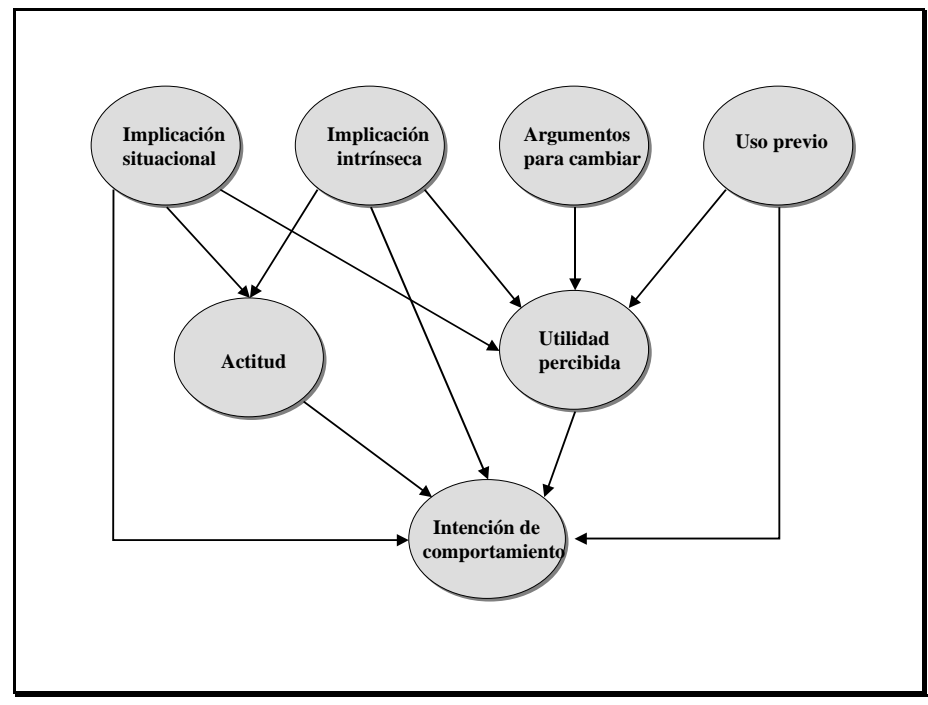

Fuente: Jackson, Chow y Leitch (1997)

Indagando en la relación entre la implicación y el TAM, Latour et al. (2002) establecen un modelo que genera cierta curiosidad, donde las variables más identificativas del TAM -utilidad percibida y facilidad de uso percibida-, se utilizan como predictoras de la implicación. Esto conlleva, lógicamente, un cambio en la 
corriente causal que señala el modelo de Jackson, Chow y Leitch (1997) respecto a estas variables conceptuales.

Karahanna y Straub (1999) tratan de determinar los antecedentes de los constructos utilidad percibida y facilidad de uso percibida del modelo TAM. Para ello añaden cuatro variables en el modelo: presencia social (capacidad para transmitir información de un modo no verbal); influencia social (normas sociales); accesibilidad percibida (acceso a la tecnología y habilidad en su manejo); y disponibilidad de entrenamiento y apoyo al usuario (ver figura 7). Las hipótesis propuestas por estos autores consiguen un considerable sustento estadístico, salvo aquéllas que están relacionadas con la variable disponibilidad de entrenamiento y apoyo, que no logran un nivel de significación aceptable.

Figura 7: Modelo de Karahanna y Strauss (1999)

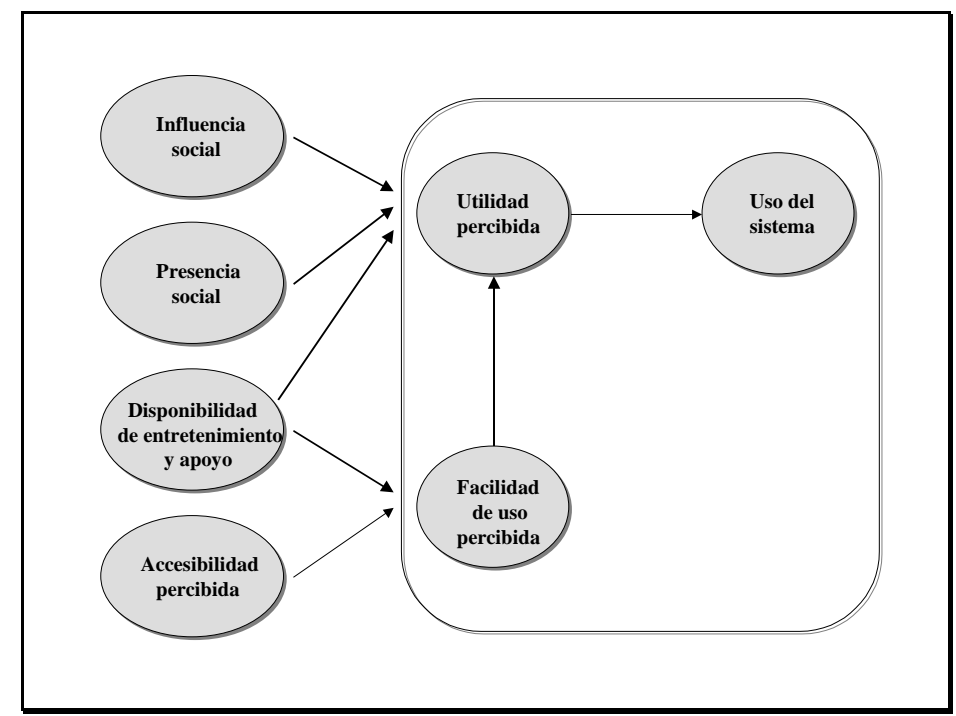

Fuente: Karahanna y Straub (1999)

Venkatesh y Davis (2000) desarrollan el modelo denominado TAM2, que pretende conciliar, en parte, la Teoría de la Acción Razonada de la Psicología Social (v. gr., FISHBEIN; AZJEN, 1980), la de la Motivación en el Trabajo (v. gr., VROM, 1964) y la de la Imagen, procedente esta última del área de la Teoría de la Decisión de Comportamiento (v. gr., BEACH; MITCHELL, 1978). El núcleo del nuevo modelo es lógicamente el TAM, buscándose a partir de él las variables que pueden tener influencia sobre las intenciones de uso, bien directamente o a través de su efecto en la utilidad percibida (ver figura 8). 
Figura 8: Extensión del Modelo de Aceptación de la Tecnología (TAM2)

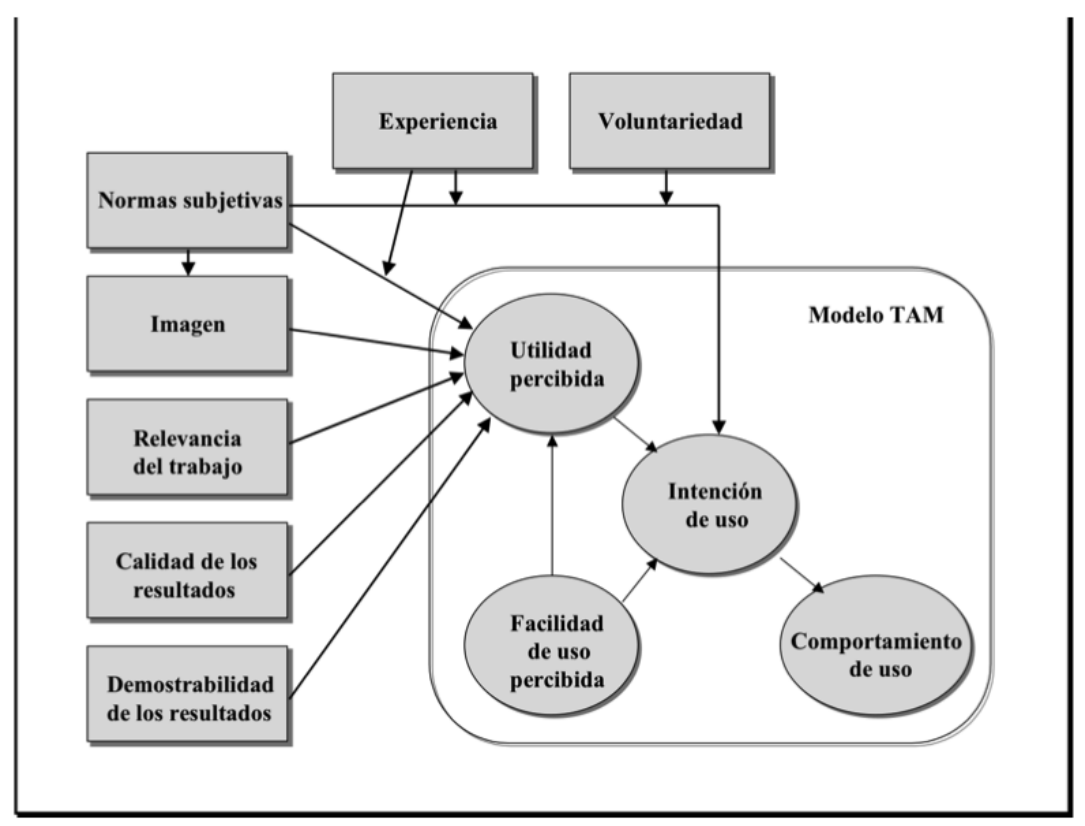

Fonte: Venkatesh y Davis (2000)

Los antecedentes se dividen en dos grupos: los procesos de influencia social (normas subjetivas, voluntariedad, imagen y experiencia) y los procesos cognitivos (relevancia del trabajo, calidad del resultado y demostrabilidad del resultado). Siguiendo a Hartwick y Barki (1994), se piensa que las normas subjetivas tienen un efecto significativo sobre la intención de uso sólo en situaciones de obediencia o sumisión, pero no en aquéllas en las que el usuario es libre para elegir; de ahí la importancia que juega la voluntariedad, entendida como el grado en el que un adoptante potencial percibe que su decisión no es el cumplimiento de una orden (AGARWAL; PRASAD, 1997a; HARTWICK; BARKI, 1994; MOORE; BENBASAT, 1991). La imagen es entendida de la misma manera que lo hacen Moore y Benbasat (1991). Respecto al constructo experiencia, el TAM2 sostiene que el efecto directo de las normas subjetivas sobre las intenciones será más fuerte antes de la implantación de la innovación y durante el inicio de su uso, pero decaerá a medida que a lo largo del tiempo se incremente la experiencia con el sistema. La variable relevancia del trabajo, denominada por Goodhue (1995) como ajuste de la tecnología a la tarea, hace referencia al grado en el que el sistema es aplicable a las labores del usuario, mientras que la calidad del resultado se refiere a la eficacia del sistema al realizar estas tareas. Por último, la demostrabilidad del resultado fue definida por Moore y Benbasat (1991) como la tangibilidad de los beneficios obtenidos al usar la innovación.

Tan sólo hemos detallado algunos modelos derivados del TAM que han tenido un mayor calado, pero existen otros muchos que han aparecido más recientemente, entre los que podemos citar el Modelo de Uso de la Tecnología en Colaboración (DENNIS; VENKATESH; RAMESH, 2003), la Teoría Unificada de la Aceptación y Uso de la Tecnología (VENKATESH et al., 2003), el Modelo Ampliado del TAM con la Teoría de la Difusión de Innovaciones, el Riesgo Percibido y el Coste (WU; WANG, 2004) o el Modelo de Informatización de la Persona Emprendedora (NDUBISI; GUPTA; NDUBISI, 2005). 


\section{Conclusiones}

Hay un gran número de modelos de adopción de innovaciones, que se relacionan con distintos paradigmas que se fundamentan en disciplinas como la Sociología, la Psicología Cognitiva y la Psicología Social. El presente trabajo realiza una revisión de los modelos de adopción de las tecnologías de la información desde una perspectiva de los modelos actitudinales, enraizados con la Psicología Social. La relación de modelos se ha desarrollado en el ámbito de los sistemas de información y del marketing. Se destaca la generalización del modelo TAM, cuyo fundamento se halla en la Teoría de la Acción Razonada, a partir del cual se definen otras propuestas que responden a variaciones de dicho modelo, como son el TAM2 y el TAME. Asimismo, se han descrito otros modelos, como el TAU, que intenta conciliar dos tradiciones distintas como son las teorías normativas y actitudinales; los modelos UIT y AIT, que tratan de fusionar las corrientes derivadas de las teorías de la Difusión de Innovaciones y la Teoría de la Acción Razonada.

\section{Referências bibliográficas}

AGARWAL, R.; PRASAD, J. The Role of Innovation Characteristics and Perceived Voluntariness in the Acceptance of Information Technologies. Decision Sciences, n.28 (3), p.557-582, 1997 a.

AGARWAL, R.; PRASAD, J. Targeting COBOL Programmers for C Training: The Role of Job Insecurity and Organizational Tenure. Journal of Systems Software, n. 37 (5), p.5-17, 1997b.

AGARWAL, R.; PRASAD, J. The Antecedents and Consequents of User Perceptions in Information Technology Adoption. Decision Support Systems, n.22 (1), p.15-29, 1998.

AGARWAL, R.; PRASAD, J. A Field Study of the Adoption of Software Process Innovations by Information Systems Proffesionals. IEEE Transactions on Engineering Management, n.47 (3), p.295-308, 2000.

AGARWAL, R.; PRASAD, J.; ZANINO, M.C. Training Experiences and Use Intentions: A Field Study of a Graphical User Interface. International Journal of Human-Computer Studies, n. 45, p.215-241, 1996.

AJZEN, I. From Intention to Actions: A Theory of Planned Behavior. En: J. KUHL; J. BECKMANNN (eds.). Action Control: From Cognition to Behavior. New York: Springer-Verlag, 1985. p.11-39.

AJZEN, I. The Theory of Planned Behavior. Organizational Behavior and Human Decision Processes, n.50, p.179-211, 1991.

AJZEN, I.; FISHBEIN, M. Understanding Attitudes and Predicting Social Behavior. Prentice Hall: Englewood Cliffs, 1980.

ANTÓN, C.; GUTIÉRREZ, J. Modelos Positivos de Comportamiento en Materia de Adopción de Innovaciones. Anales de Estudios Económicos y Empresariales, n. 12, p.55-83, 1997.

BAGOZZI, R.P. Attitudes, Intentions, and Behavior: A Test of Some Key Hypotheses. Journal of Personality and Social Psychology, n.41, p.607-627, 1981.

BAGOZZI, R.P. Buyer Behavior Models for Technological Products and Services. En: W. JOHNSTON; J.N. SHETH; G.L. FRAZIER (eds.). Advances in Telecommunications Management. Greenwich: JAI Press, 1990. vol.2. p.43-69. 
BAGOZZI, R.P.; VAN LOO, M.F. Motivational and Reasoned Processes in the Theory of Consumer Choice. En: R. FRANTZ; H. SINGH; J. GERBER; S. KAISH; B. GILAD (eds.). Handbook of Behavioral Economics: Behavioral Decision Making. Greenwich: JAI Press, 1991. vol.2B. p.401-438.

BAGOZZI, R.P.; WARSHAW, P.R. Trying to Consume. Journal of Consumer Research, n.17, p.127-140, 1990.

BANDURA, A. Self-Efficacy Mechanism in Human Agency. American Psychologist, n.37 (2), p.122-147, 1982.

BEACH, L.R.; MITCHELL, T.R. A Contingency Model for the Selection of Decision Strategies. Academy Management Review, n.3, p.439-449, 1978.

BEST, J. B. Psicología Cognitiva. Madrid: Paraninfo, 2001.

BETTMAN, J.R.; JONES, J.M. Formal Models of Consumer Behavior: A Conceptual Overview. Journal of Business, n.45 (4), p.544-562, 1972.

BETTMAN, J.R.; SUJAN, M. Research in Consumer Information Processing. En: M.J. HOUSTON (ed.). Review of Marketing, Chicago, p.197-235, 1987.

CRICK, F.H.; ASANUMA, H. Certain Aspect of the Anatomy and Physiology of the Cerebral Cortez. En J.L. MCCLELLAND; D.E. RUMELTHART; PDP INVESTIGATION (eds.). Parallel Distributed Processing: Explorations in the Microstructure of Cognition. Volume 2: Psychological and Biological Models. Cambridge: The MIT Press, 1986.

DABHOLKAR, P.A. Decision-Making in Consumer Trial of Technology-Based Self-Service Options: An Attitude-Based Choice Model. UMI (Dissertation) - Georgia State University, Atlanta, 1991.

DABHOLKAR, P.A. Incorporating Choice into an Attitudinal Framework: Analyzing Models of Mental Comparison Processes. Journal of Consumer Research, n.21, p.100-118, 1994.

DAVIS, F.D. Perceived Usefulness, Perceived Ease of Use, and User Acceptance of Information Technology. MIS Quarterly, n.13 (3), p.319-340, 1989.

DAVIS, F.D.; BAGOZZI, R.P.; WARSHAW, P.R. User Acceptance of User Technology: A Comparison of Two Theoretical Models. Management Science, n.35 (8), p.982-1.002, 1989.

DAVIS, F.D.; BAGOZZI, R.P.; WARSHAW, P.R. Extrinsic and Intrinsic Motivation to Use Computers in the Workplace. Journal of Applied Social Psychology, n.22 (14), p.1.111-1.132, 1992.

DECHARMS, R. Personal Causation: The Internal Affective Determinants of Behavior. New York: Academic Press, 1968.

DECI, E.L. Intrinsic Motivation, Extrinsic Reinforcement, and Inequity. Journal of Personality and Social Psychology, n.22, p.113-120, 1972.

DENNIS, A.R.; VENKATESH, V.; RAMESH, V. Adoption of Collaboration Technologies: Integrating Technology Acceptance and Collaboration Technology Research. Working Papers Series, Indiana University. Documento electrónico obtenido en: <www.indiana.edu/ isdept/research/workingpapers.html>. Acceso en: 2003.

EDWARDS, W. The Theory of Decision Making. Psychological Bulletin, n.51, p.380-417, 1954

EINHORN, H.J.; HOGARTH, R.M. Behavioral Decision Theory: Processes of Judgment and Choice. Journal of Accounting Research, n.19 (1), p.1-31, 1981. 
FISHBEIN, M.; AJZEN, I. Belief, Attitude, Intention, and Behavior: An Introduction to Theory and Research. Addison-Wesley: Reading, 1975.

FISHBEIN, M.; AJZEN, I. Predicting and Understanding Consumer Behavior: Attitude-Behavior Correspondence. En: I. AZJEN; M. FISHBEIN (eds.). Understanding Attitudes and Predicting Social Behavior. Englewood Cliffs: Prentice-Hall, 1980.

FRAMBACH, R.T.; BARKEMA, H.G.; NOOTEBOOM, B.; WEDEL, M. Adoption of service innovation in the business market: an empirical test of supply-side variables. Journal of Business Research, n.41 (2), p.161174, 1998.

FREDRICKS, A.J.; DOSSET, D.L. Attitude-Behavior Relations: A Comparison of the Fishbein-Ajzen and the Bentler-Speckart Models. Journal of Personality and Social Psychology, n.45 (3), p.501-512, 1983.

GOODHUE, D.L. Understanding the Linkage between User Evaluations and the Underlying Systems. Management Science, n.41, p.1.827-1.844, 1995.

HAGERTY, M. R.; AAKER, D.A. A Normative Model of Consumer Information Processing. Marketing Science, n.3 (3), p.227-246, 1984.

HARTWICK, J.; BARKI, H. Explaining the Role of User Participation in Information System Use. Management Science, n.40 (4), p.440-465, 1994.

HAUSER, J.R.; URBAN, G.L. A Normative Methodology for Modeling Consumer Response to Innovation. Operations Research, n.25 (4), p.579-619, 1977.

HU, P.J.; CHAU, P.Y.K.; SHENG, O.R.L.; TAM, K.Y. Examining the Technology Acceptance Model Using Physician Acceptance of Telemedicine Technology. Journal of Management Information Systems, n.16 (2), p.91-112, 1999.

IGBARIA, M. User Acceptance of Microcomputer Technology: An Empirical Test. OMEGA The International Journal of Management Science, n.21 (1), p.73-90, 1993.

JACKSON, C. M.; CHOW, S.; LEITCH, R. A. Toward an Understanding of the Behavioral Intention to Use an Information System. Decision Sciences, n.28 (2), p.357-389, 1997.

KARAHANNA, E.; STRAUB, D.W. The Psychological Origins of Perceived Usefulness and Ease-of-Use. Information and Management, n.35 (4), p.237-250, 1999.

KARAHANNA, E.; STRAUB, D.W.; CHERVANY, N. L. Information Technology Adoption across Time: A Cross-Sectional Comparison of Pre-Adoption and POst-Adoption Beliefs. MIS Quarterly, n.23 (2), p.183-203, 1999.

LACHMAN, R.; LACHMAN, J.; BUTTERFIELD, E.C. Cognitive Psychology and Information Processing: An Introduction. Hillsdale: Lawrence Erlbaum Associates, 1979.

LARCKER, D.F.; LESSIG, V.P. Perceived Usefulness of Information: A Psychometric Examination. Decision Sciences, n.11 (1), p.121-134, 1980.

LATOUR, M.S.; HANNA, J.B.; MILLER, M.D.; PITTS, R.E. Consumer Involvement with Personal Computer Technology: A Multi-Sample Analysis. American Business Review, p.1-11, 2002.

LAWLER, E.E.; PORTER, L.W. Antecedent Attitudes of Effective Managerial Performance. Organizational Behavior and Human Performance, n.2, p.122-142, 1967. 
LÓPEZ BONILLA, L.M.; LÓPEZ BONILLA, J.M. Estudio comparado de las estimaciones de dos versiones del modelo de Aceptación de la Tecnología (TAM) mediante los programas AMOS y PLS. Investigaciones Europeas de Dirección y Economía de la Empresa, vol. 12 (3), p.95-110, 2006.

MALTER, A.J. An Introduction to Embodied Cognition: Implications for Consumer Research. Advances in Consumer Research, n.23, p.272-276, 1996.

MANTEL, S.; KARDES, F. The Role of Direction of Comparison, Attribute-Based Processing in Consumer Preference. Journal of Consumer Research, n.25 (4), p.335-352, 1999.

MARTIN, D.; KIECKER, P. Parallel Processing Models of Consumer Information Processing: Their Impact on Consumer Research Methods. Advances in Consumer Research, n.17 (1), p.443-448, 1990.

MOORE, G.C.; BENBASAT, I. Development of an Instrument to Measure the Perceptions of Adopting an Information Technology Innovation. Information Systems Research, n.2 (3), p.192-222, 1991.

MOORE, G.C.; BENBASAT, I. Integrating Diffusion of Innovations and Theory of Reasoned Action Models to Predict Utilization of Information Technology by End-Users. En: K. KAUTZ; PRIES-HEJE (eds.). Diffusion and Adoption of Information Systems. London: Chapman \& Hall, 1996. p. 132-146.

NDUBISI, N.O.; GUPTA, O.K.; NDUBISI, G.C. The Moguls' Model of Computing: Integrating the Moderating Impact of Users' Person into the Technology Acceptance Model. Journal of Global Information Technology Management, n.8 (1), p.27-47, 2005.

OLSHAVSKY, R.W.; SPRENG, R.A. An Exploratory Study of the Innovation Evaluation Process. Journal of Product Innovation Management, n.13, p.324-340, 1996.

OZANNE, U.B.; CHURCHILL, G.A. Jr. Five Dimensions of the Industrial Adoption Process. Journal of Marketing Research, n.8, p.322-328, 1971.

PETTY, R.E.; CACIOPPO, J.T.; SCHUMANN, D. Central and Peripheral Routes to Advertising Effectiveness: The Moderating Role of Involvement. Journal of Consumer Research, n.10 (2), p.135-146, 1983.

ROGERS, E.M. Diffusion of Innovations. $3^{\text {rd }}$ Edition. New York: The Free Press, 1983.

ROGERS, E.M. Diffusion of Innovations. $4^{\text {th }}$ Edition. New York: The Free Press, 1995.

SHEPHERD, G.M. The Synaptic Organization of the Brain. $2^{\text {nd }}$ Edition. New York: Oxford University Press, 1979.

SHEPPARD, B.H.; HARTWICK, J.; WARSHAW, P.R. The Theory of Reasoned Action: A Meta-analysis of Past Research with Recommendations for Modifications and Future Research. Journal of Consumer Research, n.15, p.325-343, 1988.

SINHA, R.K.; CHANDRASHEKARAN, M. A split hazard model for analysing the diffusion of innovations. Journal of Marketing Research, n.29 (1), p.116-127, 1992.

SWANSON, E.B. Management Information Systems: Appreciation and Involvement. Management Science, n.21 (2), p.178-188, 1974.

TAN, M.; TEO, T.S.H. Factors Influencing the Adoption of Internet Banking. Journal of the Association for Information Systems, n.1, p.1-42, 2000. 
TAYLOR, S.; TODD, P.A. Assessing IT Usage: The Role of Prior Experience. MIS Quarterly, n.19 (4), p.561-568, 1995.

TRIANDIS, H.C. Attitude and Attitude Change. New York: John Wiley \& Sons, 1971.

TYBOUT, A.M.; HAUSER, J.R. A Marketing Audit Using a Conceptual Model of Consumer Behavior: Application and Evaluation. Journal of Marketing, n.45 (3), p.82-101, 1981.

VENKATESH, V.; DAVIS, F.D. A Theoretical Extension of the Technology Acceptance Model: Four Longitudinal Field Studies. Management Science, n.46 (2), p.186-204, 2000.

VENKATESH, V.; SPEIER, C. Computer Technology Training in the Workplace: A Longitudinal Investigation of the Effect of Mood. Organizational Behavior and Human Decision Processes, n.79, p.1-28, 1999.

VENKATESH, V.; MORRIS, M.G.; DAVIS, G.B.; DAVIS, F.D. User Acceptance of Information Technology: toward a Unified View. MIS Quarterly, n.27 (3), p.425-478, 2003.

VROOM, V. Work and Motivation. New York: Wiley, 1964.

WARSHAW, P.R. Buying a Gift: Product Price Moderation of Social Normative Influences on Gift Purchase Intentions. Personality and Social Psychology Bulletin, n.6, p.143-148, 1980.

WARSHAW, P.R.; DAVIS, F.D. Self-Understanding and the Accuracy of Behavioral Expectations. Personality and Social Psychology Bulletin, n.10, p.111-118, 1984.

WU, J.H.; WANG, S.C. What Drive Mobile Commerce? An Emprical Evaluation of the Revised Technology Acceptance Model. Information \& Management, n.42 (5), p.719-729, 2004.

ZAICHOWSKY, J.L. Measuring the Involvement Construct", Journal of Consumer Research, n.12 (3), p.341$352,1985$.

ZAJONC, R.B.; MARKUS, H. Affective and Cognitive Factors in Preferences. Journal of Consumer Research, n.9, p.123-131, 1982.

ZHANG, S.; MARKMAN, A.B. Overcoming the Early Entrant Advantage: The Role of Alignable and Nonalignable Differences Journal of Marketing Research, n.35, p.413-426, 1998.

ZHANG, S.; MARKMAN, A.B. Processing Product Unique Features: Alignability and Involvement in Preference Construction. Journal of Consumer Psychology, n.11 (1), p.13-27, 2001.

ZMUD, R. W. An Empirical Investigation of the Dimensionality of the Concept of Information. Decision Sciences, n.9 (2), p.187-195, 1978. 